\title{
Experimental Identification of Overall Structural Damping of System
}

\author{
Goran Radoičić - Miomir Jovanović* \\ University of Niš, Faculty of Mechanical Engineering, Serbia
}

The dynamic behaviour of large and complex structures largely depends on damping resistance in the structure. A portion of the structural energy is lost to deformations in material, friction between the contact surfaces, and relative motion within the structure. Often, in an analysis of numerical models, before the dynamic analysis of transient events (transient analysis), the damping resistance is adopted on the basis of recommendations, which implies an error of transient response (introduced by frequencies, logarithmic decrements and maximal amplitudes). Decreasing amortized vibratory movement is dependent on the extent of the structural damping. This paper presents the importance of structural damping in structural analysis and shows the experimental and theoretical procedure for identifying $G$ values of the structural damping coefficient. A model for determining the G coefficient is shown in the example of a real tower crane structure. The experimentally obtained values were then used in the transient numerical FEM analysis, as the basis for adopting the conclusions about the dynamic behaviour of this class of structures (transportation machines). The effect of the external perturbation force of trapezoidal impulse form (lifting and quickly lowering of load) is introduced and the dynamic task, as an example of the use of the G coefficient $\mathrm{G}$, is solved. The experimentally determined damping (theoretically isolated for tall truss structures) can be used in similar transient analyses.

Keywords: tower crane, truss structure, structural damping, transient response, large amplitude

\section{O INTRODUCTION}

Building sites with large construction machinery structures are very numerous and widespread today. On a single building site there is often a significant number of tower cranes, indicating the importance of this class of construction machinery. Permanent exposure to natural forces (strong winds, earthquakes, etc.) and combinations of working conditions increase the risk of a possible incident. An understanding of the dynamic behaviour of the support structures for such machinery will minimize the negative impact caused by improper handling, random events and force majeure.

The support structures of cranes are tall and heavy, and they can be defined as complex truss structures due to different shapes and properties of the elements. The structure and behaviour of the representatives of this great class of construction machinery are the subjects of current studies, [1] and [2]. The connections between various elements, held together by various methods (riveting, bolted joint and welding), also affect the complexity of the structure. Prior to dynamic analysis, it is very important to perform quality modelling of the complex structure [3]. The Finite Element Method (FEM) can be successfully used to provide a model of the complexity of the support structure. The FEM model allows us to define the geometrical and material properties of a structure very well using a large number of finite elements. The model includes all significant impacts, which define the loads, constraints and connections between elements.
The choice of damping model introduces the two damping coefficients, viscous and structural, and plays a key role in dynamic modelling. The viscous damping force is proportional to the velocity. The coefficient of proportionality is named the viscous damping coefficient. This coefficient is determined by defining the material properties of the elements forming the model for numerical analysis. The structural damping force is proportional to displacement. Structural damping includes: damping in a material of a structure, losses due to friction between contact areas, and dissipation in medium due to relative motion within the system [4]. The structural damping, thus, essentially covers the entire mechanical system (structure). The damping force is only shown through structural damping in [5]. The introduction of structural damping in the numerical analysis is estimated by the assumed coefficient $G$, [6] and [7].

The influence of damping resistance on the transient response of the mechanical structure is significant and difficult to determine accurately. Problems of identification of damping resistance and evaluation of impacts of this resistance on the dynamic response of mechanical model structures are still very current, [8] to [11].

A number of researchers introduce the "hysteretic" damping which includes a delay of elastic structure in returning to the previous position after the end of the effect of excitation force [12]. The viscous damping matrix is then proportional to the "hysteretic" damping matrix, where the coefficient of proportionality is inverse to the absolute value of 
circular frequency $\omega$ at which vibrating motion is carried.

According to the recent literature, aerodynamic damping is also used in dynamic analysis of the tall truss structures [13]. This type of damping of truss structure vibrations occurs with the motion of wind in the direction of the longitudinal axis of the tower. The increment of the average value of wind velocity increases the aerodynamic component of damping. Thus, the effect of the aerodynamic damping on vibratory movement of the structure becomes more expressed and closer to the impact of structural damping.

The impact of structural damping on tall and elastic structures with large dimensions and more degrees of freedom is particularly explored in the area of civil engineering, earthquake engineering, and structural dynamics.

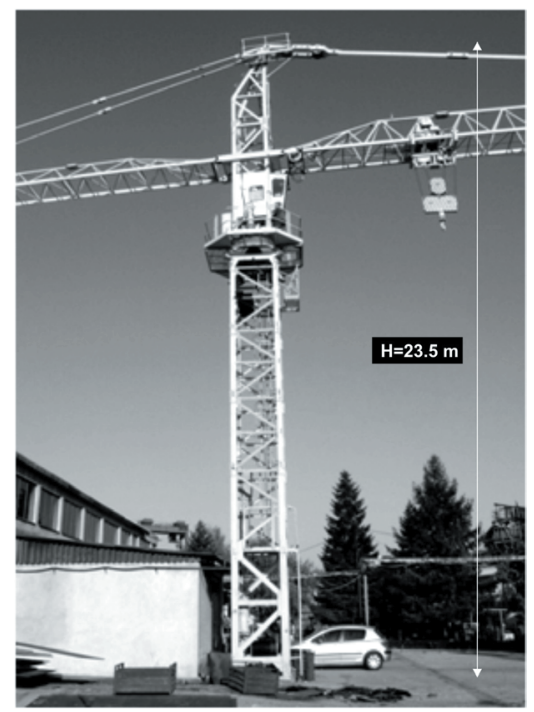

Fig. 1. Tower crane POTAIN 744e

The overall structural damping can be determined experimentally for certain eigenvalues of vibrations. The aim of this paper is to determine a single value that corresponds to the experiment on the basis of a group of several structural damping coefficients. In other words, a parallel observation of free vibrations of the structure for different values of the structural damping coefficient $G$ in numerical models enables the determination of the real structural damping on basis of the experiment. For this purpose, we can introduce a set of deformation ratios $R_{i}$ (experimentally determined) that realistically show the influence of the coefficient $G$ on the damping of free vibrations. Parameter $R_{i}$ represents the ratio of the maximal displacement amplitude $\left(U_{0}\right.$-initial $)$ and $2 i^{\text {th }}$ displacement amplitude (same phase) of an selected element of the structure for harmonic laws of vibrations, or:

$$
R_{i}=\frac{U_{0}}{U_{2 i}} ; \quad(i=1,2, \ldots)
$$

This paper shows, in an original example, the determination and influence of the structural damping coefficient on the dynamic behaviour of the structure represented by the tower crane in Fig. 1 .

\section{THEORETICAL BASIS FOR THE IDENTIFICATION OF STRUCTURAL DAMPING COEFFICIENT G}

Complex structures of different materials and shapes represent vibratory systems where some portion of energy is lost in the damping of motion (dissipation). Damping resistance occurs within the material (viscous damping), as well as within the mechanical structure of the vibratory system (structural damping). The influence of the damping resistance on the dynamic behaviour of a complex system, such as the truss structure shown in Fig. 1, is expressed in the dampened vibrating motion. The search for the dynamic response of a structure can, theoretically, be performed numerically by using FEM with appropriate software support [6] and [7]. Defining conditions for the execution of the dynamic analysis by the direct transient method, [6] and [7], requires the introduction of a number of necessary values, one of which is the overall structural damping coefficient $G$. The dynamic response of the Multiple DegreeOf-Freedom (MDOF) model is required by direct numerical integration of the system of differential equations with the elementary matrix form [14]:

$$
[M]\{\ddot{u}(t)\}+[B]\{\dot{u}(t)\}+[K T]\{u(t)\}=\{p(t)\},
$$

where $[M]$ is the inertial mass matrix, $[B]$ is the viscous damping matrix, $\left[K_{T}\right]$ is the complex stiffness matrix which includes complex terms, $\{u(t)\}$ is the displacement vector, $\{\dot{u}(t)\}$ is the velocity vector, $\{\ddot{u}(t)\}$ is the acceleration vector, and $\{p(t)\}$ is the vector of external generalized forces.

The solution of the system equations (Eq. (2)) is the displacement vector $\{u(t)\}$ and it represents the basic response of the crane structure to the effect of external forces. By differentiating the values of the displacements in time, other transient responses are also obtained (velocities, accelerations).

Structural damping is included in the equations of motion as complex terms in the stiffness matrix, 
[4], [14] and [15]. Dynamic analysis, executed by application software [6] and [7], defines the use of material damping $G_{E}$ (MATi) or the coefficient $G$ (PARAM, G) to get the overall structural damping. After the addition of the abovementioned complex terms, the complex stiffness matrix takes on the following form:

$$
\left[K_{T}\right]=(1+i G)[K]+i \sum G_{E}\left[K_{E}\right],
$$

where $G$ is the overall structural damping coefficient, $G_{E}$ is the element structural damping coefficient, $[K]$ is the global stiffness matrix, and $\left[K_{E}\right]$ is the element stiffness matrix.

Since the transient response analysis, [6] and [7], does not include complex numbers in the calculation procedure, the conversion of structural damping $G$, as well as $G_{E}$, into an equivalent viscous damping should be performed [14]. This conversion is done using Eq. (4), which is further used in the transient analysis:

$$
\left[B_{T}\right]=[B]+\frac{G}{\omega_{3}}[K]+\frac{1}{\omega_{4}} \sum G_{E}\left[K_{E}\right]
$$

where $\left[B_{T}\right]$ is the complex damping matrix, $[B]$ is the viscous damping matrix, and $\omega_{3}$ and $\omega_{4}$ are the dominant circular frequencies in radians per unit time.

Matrix $[B]$ and matrix $[K]$ are known values, obtained in the modelling process when elementary materials and the geometry of the FEM model are defined (property).

The dominant circular frequencies $\omega_{3}$ and $\omega_{4}$ are user-provided parameters, which structural analysts take from the modal analysis. Usually, these are lower frequencies of dominant dynamic states. Transient response uses various dominant modal frequencies, therefore, the software approximation (Eq. (4)) implies the use of the two modal frequencies closest to the dominant dynamic behaviour, according to [14]. The exclusion of these two parameters $\left(\omega_{3}=\omega_{4}=0\right)$ would lead to a complete neglect of the structural damping impact and thus the approximation (Eq. (4)) would represent only viscous damping, according to [6]. From the software point of view, the values of these frequencies $\left(\omega_{3}, \omega_{4}\right)$ are not consecutive modal values, but they rather represent the frequencies that correspond to the type of transient analysis (dynamic behaviour in the propagation direction). In this case study, the vibratory states of the structure in the vertical global plane are dominant, and the mode shapes that these states describe by the lowest frequency $\omega_{3}$, as well as $\omega_{4}$, are selected.
The values of $G_{E}$ are obtained by introducing the materials into the analysis procedure (GE on the MATi entry, [6] and [7]), while $G$ represents an unknown value of structural damping that needs to be defined prior to the transient analysis (PARAM, G).

In order to obtain an accurate transient response of the structure to harmonic excitations of the same eigenvalue, $\omega^{2}$, it is first necessary to experimentally determine the value of the structural damping coefficient $G$. The determination of parameter $G$ is performed by calculating the logarithmic decrement of the vibrating motion. The logarithmic decrement $\Delta$, or the decrease in declining vibratory movement [16], can be calculated as:

$$
\Delta=\ln \frac{\left|u_{n}\right|}{\left|u_{n+1}\right|}=\delta \frac{T}{2},
$$

where $\left|u_{n}\right|$ and $\left|u_{n+1}\right|$ are the absolute values of successive amplitudes, and $d$ is the damping coefficient.

In addition, an alternative logarithmic decrement $\Delta^{\prime}$ can be calculated using the following equation:

$$
\Delta^{\prime}=\ln \frac{|u(t)|}{|u(t+T)|}=\delta T,
$$

where the values $|u(t)|$ and $|u(t+T)|$ are related to the two same-phase amplitudes at the distance of one period of damped vibratory movement $T$.

Overall structural damping depends on the coefficient $\delta(G=f(d))$ which can be obtained experimentally, after determining the values of logarithmic decrement $\Delta$ (or $\Delta^{\prime}$ ) of the declining vibrating motion and the period of damped vibratory movement $T$ [16], or:

$$
\delta=\frac{2 \Delta}{T}=\frac{\Delta^{\prime}}{T}=\frac{1}{T} \ln \frac{|U(t)|}{|U(t+T)|} .
$$

\section{THE EXPERIMENTAL-THEORETICAL MODEL FOR IDENTIFICATION OF COEFFICIENT G - AN EXAMPLE}

The dynamic response of a large truss structure on the effect of the external disturbance force (due to a sudden lowering of load to the ground) is required in the experiment. The tower crane P744e in Fig. 1 was selected as an object of examination to perform the experiment. 
The foundation of the crane pylon was composed of reinforced concrete and was located on ground with a compressibility class II. The base of the crane was supported only elastically and defined by the basic stiffness of the ground. The measurement of local movement of the base in relation to the surrounding ground under the working loads showed insignificantly minor values. The trial load was 5.3 or $2.65 \mathrm{t}$ in the range of 20.88 or $45 \mathrm{~m}$, and it was slightly larger than the nominal value for causing vibrations of larger amplitudes. Special attention was directed to preserving the stability of the crane and the stressstrain state in the field of elasticity.

The following values were measured experimentally: a) deformations at the root of the pylon on opposite sides using two strain gauges; b) change of force on the hook using a force transducer; c) deflection of the boom in the vertical plane for two characteristic ranges (20.88 and $45 \mathrm{~m}$ ) using a pen on a vertical plotter (Fig. 2).

On this occasion, the following HBM measuring equipment was used [17]: an MGC Plus measuring amplifier, an LY 10/120W strain gauge, and a U2A $10 \mathrm{t}$ dynamometer. The measuring results were processed using HBM Catman software.The testing was performed according to the following scenario: 1) the trial load was hoisted to the maximal height and stopped; 2) the next step was the calming of the load in order to dampen the vibrations of the crane caused by hoisting; 3 ) finally, by suddenly lowering the load until striking the ground, unloading was caused, along with the free damped vibrations of the entire structure.

Fig. 3 shows the results of vibrating motion for two opposite referent points of the root structure of the tower crane, measured by strain gauges. The abscissa shows the time of the experiment in seconds, and the ordinate shows the values of the measured deformations of considered structural elements in $\mu \mathrm{m} / \mathrm{m}$. Dynamically, the most interesting period occurs after sudden unloading of the structure, when maximal amplitudes are also observed. That is why the diagram in Fig. 3 does not contain parts of the vibratory movement that are related to the initial period of the experiment that lasted for 50 sec (hoisting and calming). The lighter curve MG1 is related to the measuring gauge at the front side the side of the load, and the darker curve MG2 to the measuring gauge at the opposite side of the pylon. The maximal value of the micro-deformations is a little over $100 \mu \mathrm{m} / \mathrm{m}$, which is within the boundaries of elasticity.

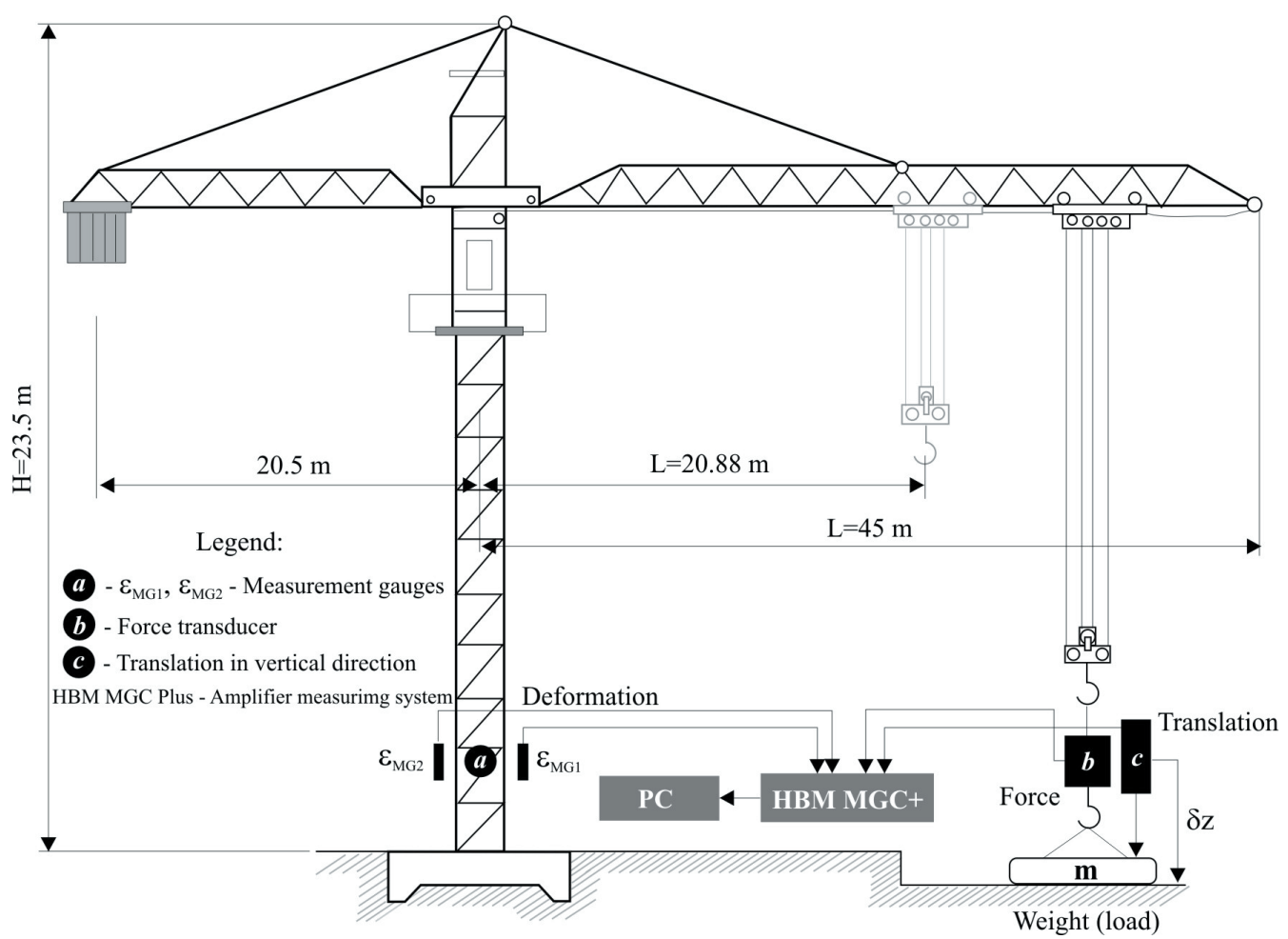

Fig. 2. The layout of the measuring system on the tower crane $P 744 \mathrm{e}$ 


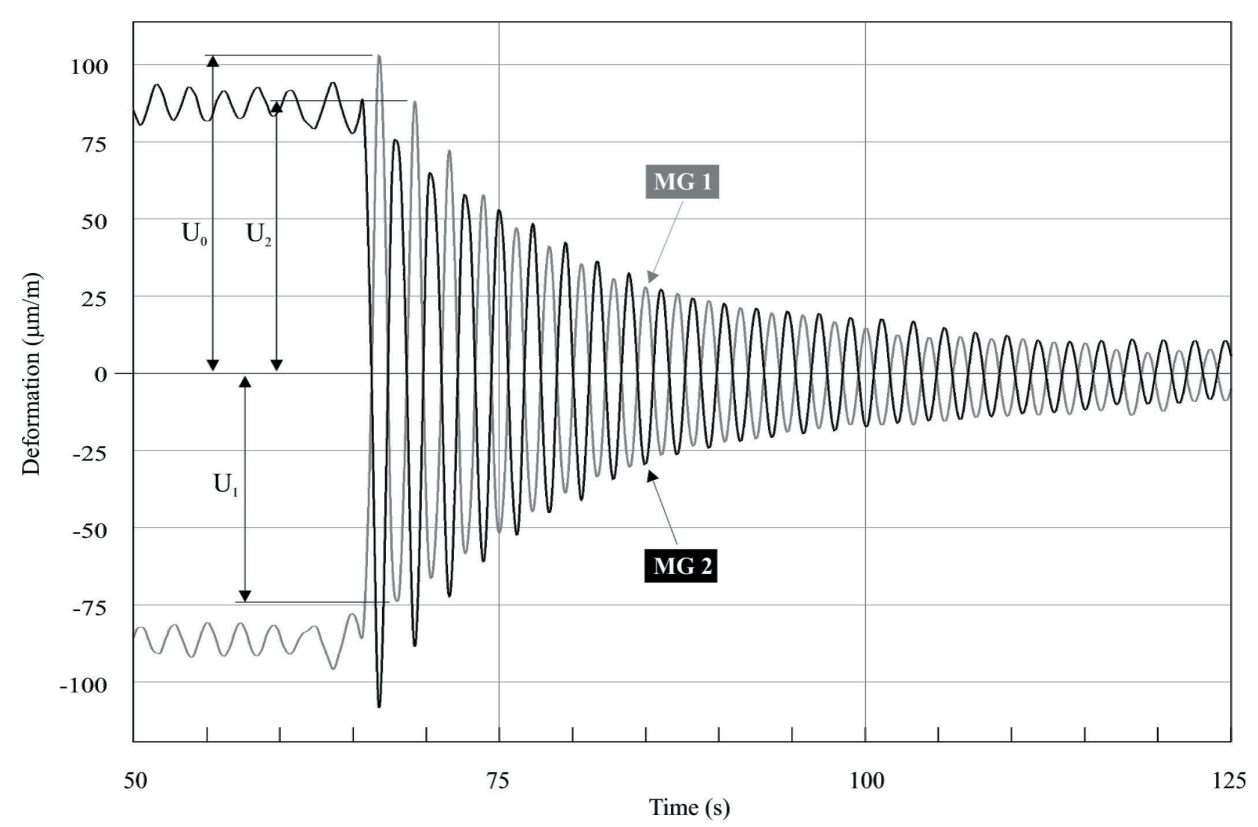

Fig. 3. Micro-dilatations ( $\varepsilon M G 1, \varepsilon M G 2$ ) of the tower root where measuring gauges MG1 and MG2 were placed

The decreased vibrating motion was characterized by a changeable period of vibration (insignificant differences), thus the structural damping coefficient should be examined for an optimal number of logarithmic decrements and appropriate periods of vibrations. In the research measurements, the determination of the logarithmic decrement D (or D') is more reliable if the number of measurements of the same mode of vibration $(w)$ is larger. In the presented experiment, twenty-four deformation amplitude changes $e$ were found before the structural calm, after which the deformation vibration level fell below $8 \%$, and they were not observed further. A wider range of measured deformations introduces random influences that generate non-regular vibrations. Areas with regular vibrations are necessary for the study ("proper" vibration without noise). Even the choice of measuring gauge and measuring technology was performed to ensure a better and purer signal in relation to other types of physical measuring values (accelerations). Therefore, the zones of relatively weak signals (small deformations) with a relatively greater presence of random effects were avoided.

Substituting the variables for the amplitudes shown in Fig. 3 into Eq. (7), the general expression used to calculate the particular values of the damping coefficient can be written as:

$$
\delta_{i}=\frac{1}{T_{i}} \ln \frac{\left|U_{2(i-1)}\right|}{\left|U_{2 i}\right|} ; \quad i=1,2, \ldots,
$$

where $U_{2 i}$ is the amplitude of displacement and $U_{2(i-1)}$ is the previous same-phase amplitude of displacement at the distance of one period of vibration $T_{i}$.

Fig. 4 shows the results of the experimental determination of the damping coefficient according to Eq. (8). The discrete values of the logarithmic decrements and the periods of vibrations from the moment of unloading the structure to the end of the experimental test $\left(t_{\max }=120 \mathrm{~s}\right)$ are introduced. The measured values of the damping coefficient $d$ are shown with crosses. The third-degree polynomial approximation (solid line) shows the change in this coefficient in the discrete time calculations.

The diagram in Fig. 4 suggests the nonlinear nature of the structural damping in complex structures, i.e. the damping coefficient does not represent a constant default value in numerical analyses, which is caused by the change in the vibrating mass of the system (rejection of load). In this experiment, the damping coefficient takes two extreme values: $\delta_{\min }=0.004$ and $\delta_{\max }=0.108$, which are specific to the very end of the damped vibration measurement.

Greater dissipation of coefficient $d$ (Eq. (8)) always occurs in experimental recordings at the end of the vibration process. If the observation area is narrowed, then the propagation damping area is also narrower (due to a smaller number of observed amplitudes). Therefore, the measured results were statistically processed and the mean value of damping $\bar{\delta}=0.050853$ (Eq. (9)), as well as the empirical 


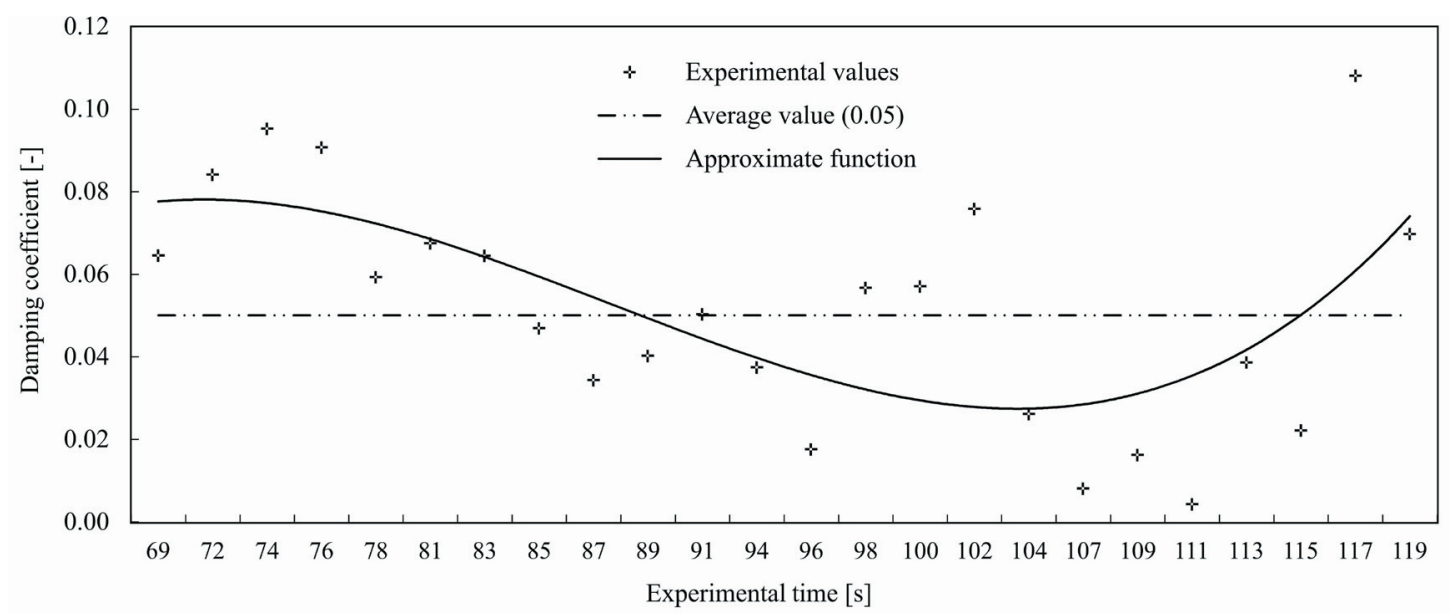

Fig. 4. Change of the damping coefficient d, dependent on the logarithmic decrement $\Delta^{\prime}$

variance of the statistical set $\operatorname{Var}(d)^{\prime \prime}=0.00079131$ (Eq. (10)), were obtained.

$$
\begin{gathered}
\bar{\delta}=\frac{1}{n} \sum_{i=1}^{n} \frac{1}{T_{i}} \ln \frac{U_{2(i-1)}}{U_{2 i}}, \quad n=24, \\
\operatorname{Var}(\delta)=\frac{1}{n-1} \sum_{i=1}^{n}\left(\delta_{i}-\bar{\delta}\right)^{2} .
\end{gathered}
$$

If Eq. (8) is calculated for more same-phase amplitudes, then the dissipation is smaller. Regardless of the dissipation in Fig. 4, the calming process in Fig. 3 (obtained by measurement) is very orderly and asymptotically damped in accordance with the theory (the tops of the amplitudes are located on the envelopes).For the transient analysis that follows, the nonlinear nature of the structural damping should be represented as linear. The problem is simplified by introducing into the calculation the rounded average value of the measured results of the damping coefficient $\delta_{\exp }=\bar{\delta}=0.05$ (Eq. (9)) at the selected measuring point of the tower crane P744e (Fig. 4, the long dash-dot-dot line).

\section{MODEL APPLICATION}

The application of the previously obtained experimental results in dynamic analyses and the impact of the overall structural damping on the transient response of the structure are shown in an example of direct transient analysis in the FEM model of the tower crane. The transient response of the structure of the tower crane P744e is calculated numerically by using FEM in the previously developed model with the following general characteristics: model mass $M_{M O D}=72204 \mathrm{~kg}$, number of finite elements $N_{E L E M E N T}=1667$, number of nodes $N_{N O D E}=1146$, overall number of degrees of freedom $N_{D O F}=6876$.

The dynamic behaviour of the structure is caused by the external force that occurs during the sudden unloading of the structure due to an unexpected, highspeed lowering of the load. This can be observed as an incident (for example, the falling of a load). Structural calming, after hoisting the load, is achieved by choosing the optimal calming time. The new vibrating motion is excited by the force of the sudden unloading during the lowering of the load to the ground. The duration of unloading is taken from the experiment and it is $\Delta t=0.83 \mathrm{~s}$ (the diagram in Fig. 5).

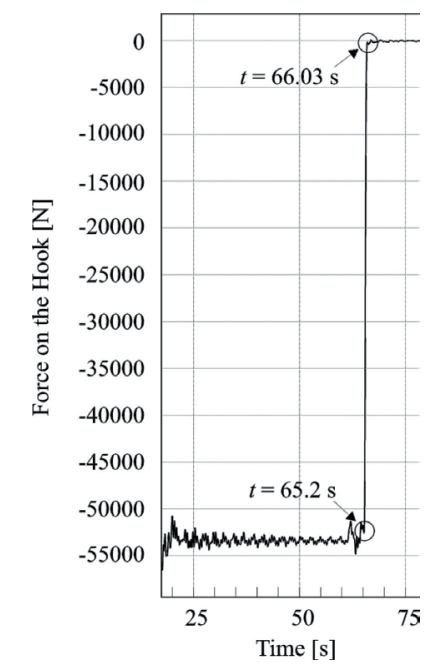

Fig. 5. The measured changes in force on the hook

The theoretical perturbation force has a trapezoid impulse form [18], as shown in Fig. 6. To numerically 
solve the dynamic problem of free damped vibrations of the system, the MDOF application software, [6] and [7], is used.

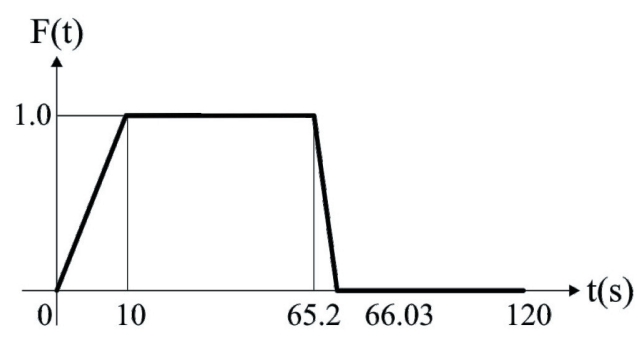

Fig. 6. Impulse shape force

The significant circular frequencies of the numerical model were determined by modal analysis of the first one hundred eigenvalues. The basic circular frequency $\omega_{1, m}=0.1687 \mathrm{~Hz}$ is specific for the rotation around the main vertical axis of the crane pylon. This frequency is not significant for the transient analysis that is performed in this application. The dominant vibratory movement occurs in the vertical plane during the lowering/falling of load. The circular frequencies in the second and the fourth vibration mode $\left(\omega_{2, m}=0.4749 \mathrm{~Hz}, \omega_{4, m}=1.5444\right.$ $\mathrm{Hz}$ ) best represent the structural vibrating nature in the vertical plane, and they are further used in the transient analysis (Eq. (4)). Fig. 7 shows the second (Mode 2), the fourth (Mode 4), and the sixth (Mode 6) eigenvalue, i.e. the vibration mode of the developed FEM model.

Matrices of mass, stiffness, and viscous damping are determined on the basis of the selected materials and the geometry of the model in the modelling process. The best value of structural damping from the experiment was adopted and converted into the value of viscous damping in Eq. (4) by using two specific circular frequencies $\left(\omega_{3}=\omega_{2, m} ; \omega_{4}=\omega_{4, m}\right)$, which were obtained in the modal analysis. By numerical integration of the system equations in Eq. (2) in the direct transient analysis, node displacements $u(t)$ were obtained for the model that freely vibrates after unloading.

Apart from determining the transient response using the structural damping coefficient $G=0.05=$ $G_{\text {exp }}$, other transient responses of the model were also numerically determined. The behaviour of the numerical model under the influence of the same load set was observed, i.e. the same conditions of unloading only at different values of the structural damping of the system. The overall structural damping coefficient $G$, in numerical simulations, corresponded to the coefficient $\delta$, and it has taken values ranged from $G=0.01$ to 0.108 . The abovementioned damping coefficients were functionally dependent $G=f(d)$.

It is difficult to examine internal losses (mathematical dependencies) in the structural damping coefficient $G$ at the level of this complex system, and the total presence of all internal influences, according to the Eq. (3), can only be experimentally determined. The character of the connection between $G$ and $\delta$ depends on the particular construction of structural elements and their mutual contacts.

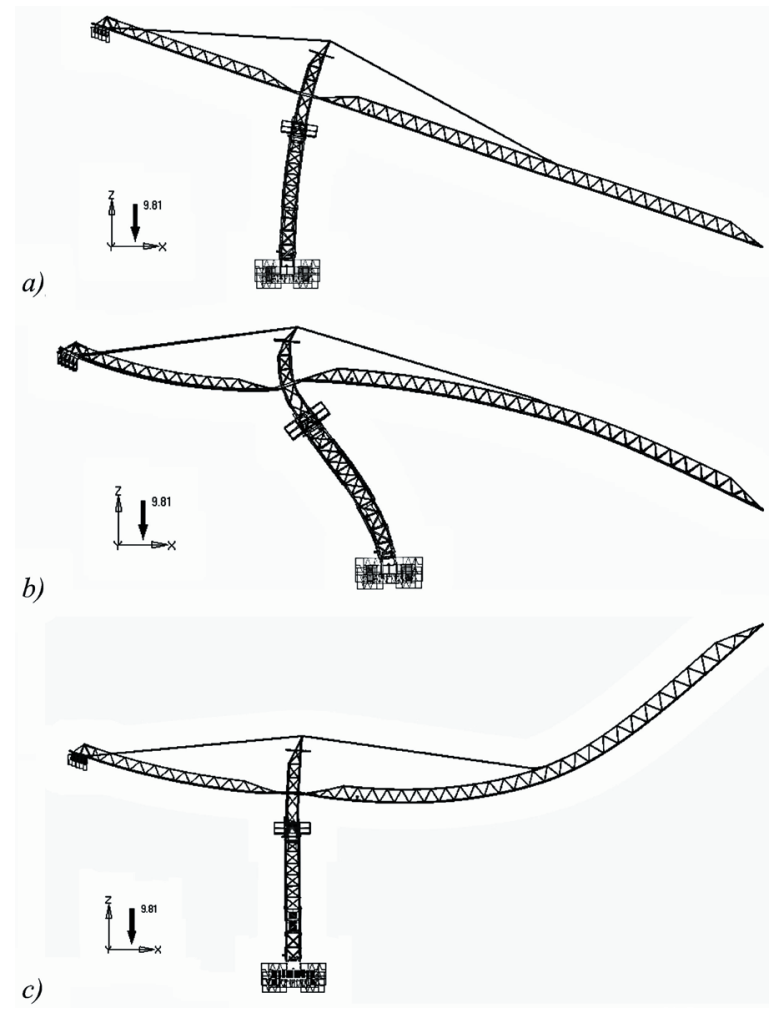

Fig. 7. Eigenvalues of vibrations; a) Mode 2, forward-back $\left(\omega_{2, m}=0.4749 \mathrm{~Hz}\right)$, b) Mode 4, forward-back $\left(w_{4, m}=1.5444 \mathrm{~Hz}\right)$, c) Mode 6, up-down $\left(w_{6, m}=2.0274 \mathrm{~Hz}\right)$

For the given values of the coefficient $G$, the ratios of numerically determined amplitudes $R_{i}$ were obtained according to Eq. (1). The obtained ratios $R_{i}$ were grouped in curves according to the value of the constant $G$. The curves of changes in the numerical amplitude ratios $R_{i}$ are shown in Fig. 8 , and they represent the appropriate mathematical approximations in exponential form, obtained numerically. The same figure shows the values of the ratio $R_{i}$, obtained experimentally, with crosses. The abscissa shows the shortened time $t$ of the experiment/ numerical test, which covers the period from the occurrence of the initial and highest vibration $U_{0}$ 
up to the time $t=91.95 \mathrm{~s}$. This shortened overview has greater visibility and shows values of the ratio $R_{i}$ for the first 13 numerically obtained same-phase amplitudes $(i=1, \ldots, 12)$, with only a few values of the coefficient $G$.

The change in the amplitude ratio $R_{i}$, shown in the diagram (Fig. 8), is performed during the calming of the structure, yet it is not a time function but a function of position $i$ of the amplitude $U_{2 i}$ with regard to the initial amplitude $U_{0}$ (e.g. if $i=4 \Rightarrow U_{2 i}=U_{8}$ then $R_{i}=R_{4}=U_{0} / U_{8}$ ) and it can be represented using the approximate exponential form:

$$
R_{i}=C_{1} e^{C_{2} i},
$$

where $C_{1}$ and $C_{2}$ are the constants which depend on the form of the exponential function. For the experimentally obtained curve of the change in the amplitude ratio $R_{i}$, these constants take values $C_{1}=1.1882$ and $C_{2}=0.133$, thus the approximate exponential equation for the observed example can be written as:

$$
R_{i}\left(G_{\text {approx. }}\right)=1.1882 e^{0.133 i} .
$$

By increasing the value of the structural damping coefficient $G$, the amplitude ratios $R_{i}$ become more pronounced and the damping of the vibration becomes greater, making the fastest calming of the structure correspond to the largest coefficient $G=0.1$ (Fig. $8)$. The specific value of the structural damping coefficient $G$ was found by changing the different values of this coefficient in the theoretical model [6] and [7]. This characteristic value of the coefficient $G$ corresponds to real behaviour of the structure based on the experiment. The numerical approximation of damped vibration of the real structure with the appropriate structural damping coefficient $(G=0.05)$ is:

$$
R_{i}(G=0.05)=1.0002 e^{0.1568 i}
$$

\section{CONCLUSION}

In the development of numerical models for the needs of dynamic analyses of large structures, it is very difficult to precisely determine the dissipation energy in the structure. The damping matrix represents the dissipation energy, which contains a constant of the overall structural damping $G$. This matrix is used in the software to determine the dynamic response of the structure. The damping of vibrating motion, as well as the duration of calming of the structure, depends on the value of coefficient $G$. In order to accurately determine coefficient $G$, it is desirable to perform experimental dynamic tests, which are presented in this paper.

A transient analysis of the complex numerical model of the tower crane leads to conclusions as to the impact of structural damping on damped vibrations in large support structures. The results indicate that similar vibrating systems, with increasing values of the structural damping coefficient, have a shorter calming period (a faster fall in vibrations). Vibrations of such systems are dampened more quickly (Fig. 8; $G=0.07, G=0.1)$. Furthermore, it can be concluded that in weakly damped vibrations (at lower coefficients $G)$ simpler mathematical approximations for the identification of the structural damping coefficient can also be applied. Therefore, Fig. 8 shows that the exponential curve $R_{i}(G=0.02)$ can be replaced by a linear function with acceptable accuracy.

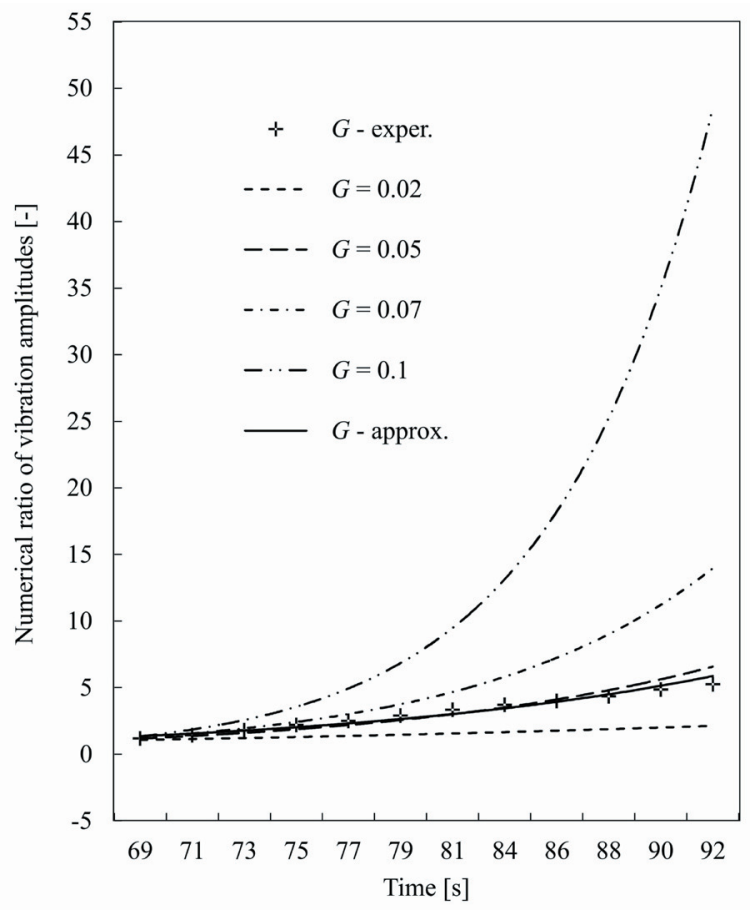

Fig. 8. The influence of the structural damping coefficient $\mathrm{G}$ on a high truss structure (numerical simulation)

Models that use experimentally determined overall structural damping $G$ are more reliable, with wide boundaries within which this ratio is changed. In the absence of experimental values, using the coefficient $G$ of similar categories and similar sizes of structures is acceptable. Experiments can give good descriptions of the initial conditions of movement, the velocities of unloading and loading of 
structures for impulse effects. In extreme cases, the observed amplitudes may belong to a class of large displacements, since they can reach up to $10 \%$ of the object's height.

The experimental-numerical model for the identification of coefficient $G$, as shown in this paper, can be applied to similar structures (larger tower cranes, portal-rotating cranes, mobile cranes, long/tall objects, pylons and towers) that vibrate with significant amplitudes under the influence of the external perturbing force. Reliable values for the structural damping coefficient for further use in similar analyses can be obtained by applying this model.

\section{ACKNOWLEDGEMENT}

The paper is a part of research carried out under project TR35049. The authors would like to thank the Ministry of Education and Science of the Republic of Serbia for their support.

\section{REFERENCES}

[1] Raubar, E., Vrančić, D. (2012). Anti-sway system for ship-to-shore cranes. Strojniški vestnik - Journal of Mechanical Engineering, vol. 58, no. 5, p. 338-344, DOI: $\quad 10.5545 / \mathrm{sv}-\mathrm{jme} .2010 .127$, DOI:10.5545/svjme.2010.127.

[2] Gašić, M., Savković, M., Bulatović, R. (2011). Optimization of trapezoidal cross section of the truck crane boom by Lagrange's multipliers and by differential evolution algorithm (DE). Strojniški vestnik - Journal of Mechanical Engineering, vol. 57, no. 4, p. 304-312, DOI:10.5545/sv-jme.2008.029.

[3] Jovanović, M., Radoičić, G., Petrović, G., Marković, D. (2011). Dynamical models quality of truss supporting structures. Facta Universitatis, Series: Mechanical Engineering, vol. 9, no. 2, p. 137-148.

[4] Gaul, L. (1999). The influence of damping on waves and vibrations. Mechanical Systems and Signal Processing, vol. 13, no. 1, p. 1-30, DOI:10.1006/ mssp.1997.0185.

[5] Bošnjak, S., Zrnić, N., Dragović, B. (2009). Dynamic response of mobile elevating work platform under wind excitation. Strojniški vestnik - Journal of Mechanical Engineering, vol. 55, no. 2, p. 104-113.

[6] MSC/Nastran v68 - Basic Dynamic Analysis (2004). MSC.Software Corporation, from: http://www. mscsoftware.com, accessed at 2004-08-26.
[7] MSC/Nastran v70 - Advanced Dynamic Analysis (2004).MSC.Software Corporation, from http://www. mscsoftware.com, accessed at 2004-08-26.

[8] Li, Q.S., Fang, J.Q., Jeary, A.P. (2000). Free vibration analysis of cantilevered tall structures under various axial loads. Engineering Structures, vol. 22, no. 5, p. 525-534, DOI:10.1016/S0141-0296(98)00124-2.

[9] Takewaki, I., Nakamura, M. (2000). Stiffness damping simultaneous identification using limited earthquake records. Earthquake Engineering and Structural Dynamics, vol. 29, no. 8, p.1219-1238, DOI:10.1002/1096-9845(200008)29:8<1219::AIDEQE968>3.0.CO;2-X.

[10] Lin, Y.Y., Miranda, E., Chang, K.C. (2005). Evaluation of damping reduction factors for estimating elastic response of structures with high damping. Earthquake Engineering and Structural Dynamics, vol. 34, no. 11, p.1427-1443, DOI:10.1002/eqe.499.

[11] Johnson, C.D., Kienholz, D.A. (1983). Prediction of damping in structures with viscoelastic materials using MSC/Nastran. MSC/Nastran Users' Conference Proceedings, paper no.17.

[12] Feriani, A., Perotti, F. (1996). The formation of viscous damping matrices for the dynamic analysis of MDOF systems. Earthquake Engineering and Structural Dynamics, vol. 25, no. 7, p. 689-709, DOI:10.1002/ (S ICI) $1096-9845$ (199607)25:7<689::A ID EQE575>3.0.CO;2-L.

[13] Holmes, J.D. (1996). Along-wind response of lattice towers - II. aerodynamic damping and deflections. Engineering Structures, vol. 18, no. 7, p. 483-488, DOI:10.1016/0141-0296(95)00131-X.

[14] Rose, T. (2002). An approach to properly account for structural damping, frequency-dependent stiffness/ damping, and to use complex matrices in transient response. Worldwide Aerospace Conference \& Technology Showcase, presentation no. 6, from:www. mscsoftware.com/events/aero2002/partner/index.cfm, accessed at 2002-04-08.

[15] Marsh, E., Slocum, A. (1996). An integrated approach to structural damping. Precision Engineering, vol. 18, no. 2-3, p. 103-109, DOI:10.1016/0141-6359(96)00051-7.

[16] Rašković, D. (1965). Theory of Vibration. Scientific Book, Belgrade.

[17] Hottinger Baldwin Messtechnik (2011). HBM, from: www.hbm.com, accessed at 2011-01-01.

[18] Prolović, V., Bonić, Z., Živković, S. (2004). Dependence of the basic dynamic parameters on the disturbing force of the impact impulse type with different forms. FactaUniversitatis, Series: Architecture and Civil Engineering, vol. 3, no. 1, p. 2331, DOI:10.2298/FUACE0401023P. 